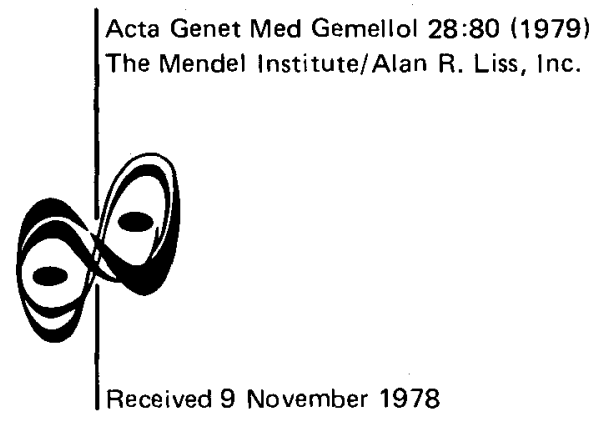

Letter to the Editor

\title{
A Reply to Chakraborty
}

\author{
Steve Selvin \\ Department of Biomedical and Environmental Health Sciences, University of California, \\ Berkeley
}

In regard to the manuscript titled "Twin Zygosity Diagnosis by Genetic Systems: An Efficiency Evaluation" by R. Chakraborty, I am not inclined to quibble about which systems are more informative or about the appropriateness of efficiency measures. However, the assumptions underlying my approach [Acta Genet Med Gemellol 26:81-82] $81-82)$ are explicitly stated:

Of course, these efficiencies will be obtained only in the case when all genotypes in the system are identified. Similar probabilities of concordance can be derived on the basis of genetic system phenotypes but no general expression is possible for all cases [all numbers of alleles].

The table of efficiences was computed under the assumption that all phenotypes are identified. Perhaps this is not an extremely realistic assumption for the ABO-system, but it is indeed necessary when the goal is to compare all genetic systems by a single measure. The fact that I did not include a discussion of the large variety of cases that arise when dominance or incomplete penetrance is present or of the cases where the genotypes are not distinguishable by present laboratory techniques should not be misleading. It is certainly not an error as Mr. Chakraborty states. 